๑ Open Access Full Text Article

\title{
Secondary Syphilis in a 12-Year-Old Girl Misdiagnosed as Pityriasis lichenoides et varioliformis acuta: A Case Report
}

\author{
Min Zhang ${ }^{1, *}$ \\ Jia $\mathrm{Yu}^{2, *}$ \\ Hui Huang' \\ Cunjian Zhou' \\ Zhifang Zhai' \\ Yi You' \\ Zhiqiang Song' \\ 'Department of Dermatology and \\ Venereology, The First Affiliated Hospital, \\ Army Medical University, Chongqing, \\ People's Republic of China; ${ }^{2}$ Department \\ of Dermatology, Distinct HealthCare, \\ Chongqing, People's Republic of China
}

*These authors contributed equally to this work
Correspondence: Zhiqiang Song Department of Dermatology and Venereology, The First Affiliated Hospital, Army Medical University, No. 30 Gaotanyan Street, Chongqing, 400038,

People's Republic of China

Tel +86-2368773025

Email zycocozm20II@qq.com

\begin{abstract}
Syphilis is a complex, systemic infectious disease caused by Treponema pallidum subspecies pallidum. Herein, we report a rare case of secondary syphilis with probable neurosyphilis that was misdiagnosed as pityriasis lichenoides et varioliformis acuta (PLEVA) in a 12-year-old human immunodeficiency virus (HIV) negative patient. A female patient presented to our hospital with a four-month history of relapsed systemic rash, accompanied by hair loss, arthralgia and fatigue. Based on physical examination and skin biopsy, she was initially diagnosed as PLEVA and treated both locally and systemically but failed to present a dermatologic improvement. The diagnosis of secondary syphilis with probable neurosyphilis was made based on serologic and cerebrospinal fluid tests. After neurosyphilis therapy, the clinical manifestations of the patient were significantly improved. Physicians should be alert for the possibility of syphilis when encountering cases with unusual clinical manifestations.
\end{abstract}

Keywords: syphilis, neurosyphilis, pityriasis lichenoides et varioliformis acuta

\section{Introduction}

Syphilis is a systemic and chronic infectious disease caused by Treponema pallidum subspecies pallidum, which can be transmitted both sexually and congenitally. In recent years, the incidence of syphilis has increased markedly around the world, making it a global public health problem.

The clinical course of syphilis can be divided into primary, secondary, latent and tertiary stage. Primary syphilis is characterized by a single painless indurated ulcer (the chancre) at the site of inoculation within 3 weeks after infection, usually accompanied by painless inguinal lymphadenopathy. The classic chancre is seen in about $60 \%$ of primary syphilis and resolves spontaneously even in untreated patients. Secondary syphilis occurs 6-8 weeks later and may not be preceded by a typical primary lesion, which hampers the diagnosis of it. Latent stage is the asymptomatic stage between the secondary and tertiary stages. About two-thirds of untreated cases remain in this stage lifelong and others progress to the tertiary stage. ${ }^{1}$

Secondary syphilis has diverse clinical presentations and thus be regarded as "a great imitator". Awareness of the presentations of secondary syphilis can facilitate the early diagnosis of this disease. Herein, we report a rare case of secondary syphilis with probable neurosyphilis that was misdiagnosed as pityriasis lichenoides et varioliformis acuta (PLEVA) in a 12-year-old human immunodeficiency virus (HIV) negative patient. 


\section{Case Presentation}

In November 2016, a 12-year-old female patient presented to our clinic with a four-month history of relapsed systemic rash, accompanied by hair loss, arthralgia and fatigue. Physical examination revealed thinning hair on the vertex; numerous scattered erythema and papules on the scalp, face, trunk and extremities; lots of hypertrophic plaques with white scales on the occipital scalp and neck. Some of the lesions showed necrosis and escharosis. Pruritus, joint tenderness and hyperpigmentation were also present (Figure 1A-F). There was no lymphadenopathy. The patient was initially diagnosed as having eczema and suspected systemic lupus erythematosus at a local hospital due to scattered erythema and papules on her face, accompanied by pruritic rash, hair loss, arthralgia and fatigue in July 2016. Four months later, she visited the Yueqing People's Hospital and was diagnosed as PLEVA and suspected systemic lupus erythematosus. After treatment with methylprednisolone tablets, celecoxib, rabeprazole, calamine lotion and potassium chloride sustained release tablets, the patient had relief from her arthralgia but failed to present a dermatologic improvement.
A skin biopsy was then performed on an erythematous lesion of the neck and an escharosis lesion of the back. H\&E staining results showed PLEVA-like histological features such as hyperkeratosis in the epidermis, mild acanthosis, elongated rete ridges, vacuolization of the basal layer with exocytosis of inflammatory cells, lymphohistiocytic infiltration around the vascular plexus and red blood cells (RBC) extravasation in the dermis (Figure 2A-D). Based on all the information obtained from physical examination, disease history inquiry and biopsy, the patient was suspected with PLEVA, psoriasis or papulonecrotic tuberculid and was hospitalized.

After hospitalization, laboratory findings revealed the patient had abnormal liver function with elevated alanine transaminase of $124.2 \mathrm{IU} / \mathrm{L}$, aspartate transaminase of 254.4 IU/L, gamma-glutamyltransferase of $88 \mathrm{IU} / \mathrm{L}$ and globulin of $44.00 \mathrm{~g} / \mathrm{L}$. Results from other examinations such as blood routine examination (white blood cells of $4.65 \times 10^{9} / \mathrm{L}$, red blood cells of $3.77 \times 10^{12} / \mathrm{L}$, hemoglobin of $111 \mathrm{~g} / \mathrm{L}$ and platelet of $\left.281 \times 10^{9} / \mathrm{L}\right)$, complement $\mathrm{C} 3(1.05 \mathrm{~g} / \mathrm{L})$ and $\mathrm{C} 4(0.16 \mathrm{~g} / \mathrm{L})$ test, anti-streptolysin $\mathrm{O}$ test $(54.60 \mathrm{IU} / \mathrm{mL})$ and rheumatoid factor test $(<20.00 \mathrm{IU} / \mathrm{mL})$ were all normal. Besides, result of the anti-extractable nuclear antigen assay was negative. The
A

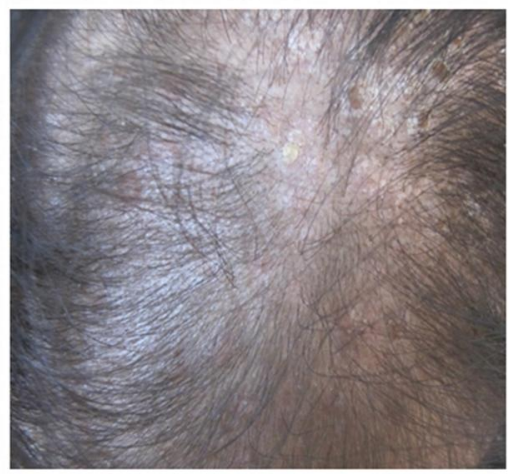

D

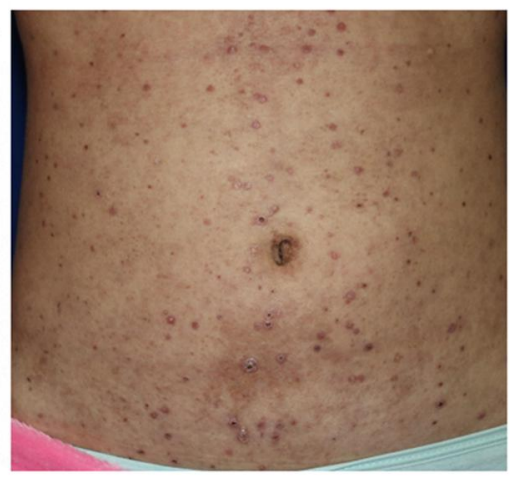

B

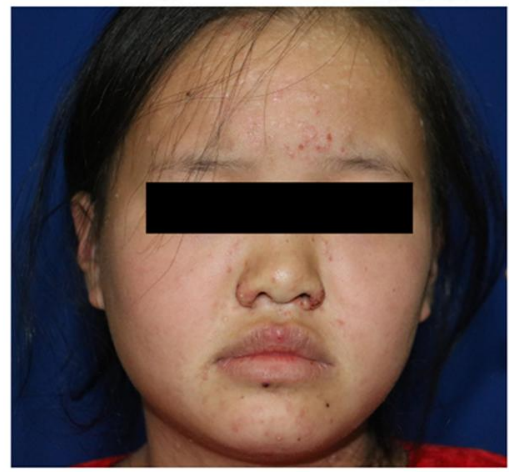

$\mathrm{E}$

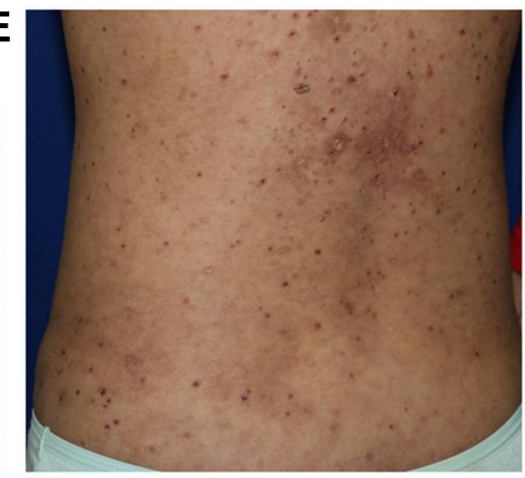

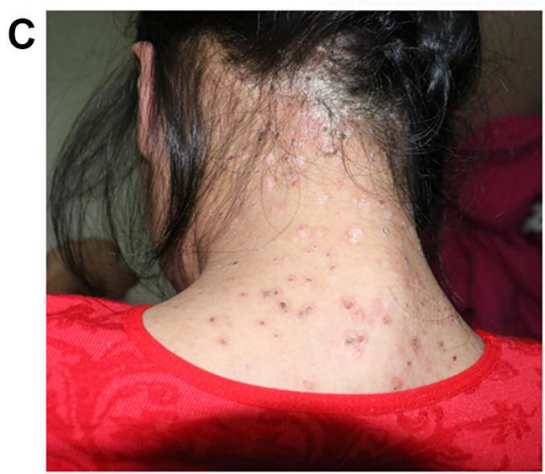

$\mathbf{F}$

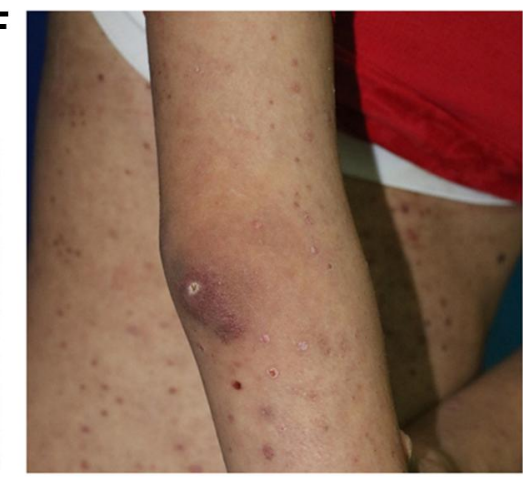

Figure I Clinical manifestations and H\&E staining before diagnosis of syphilis. (A) Thinning hair on the vertex with scattered erythema and papules on the scalp; (B) numerous scattered erythema and papules on the face; (C) lots of hypertrophic plaques with white scales on the occipital scalp and neck; (D-F) scattered erythema and papules on the trunk (D and $\mathbf{E}$ ) and extremities (F), some of the lesions showed necrosis and escharosis. Hyperpigmentation was presented. 

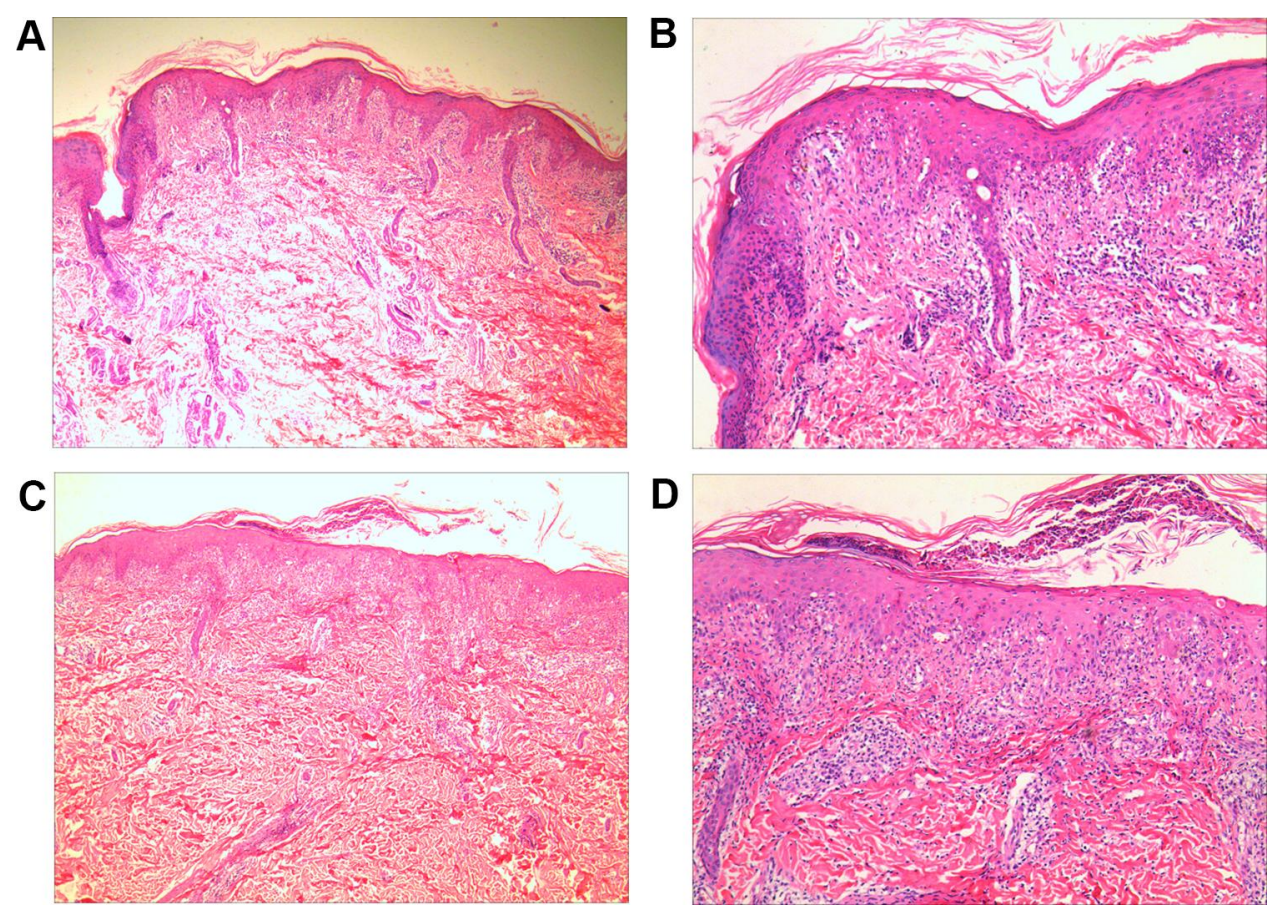

Figure 2 H\&E staining shows PLEVA-like features before diagnosis of syphilis. (A and B) H\&E staining of an erythema of the neck (A, X 40; B, X I00); (C and D) H\&E staining of an escharosis lesion of the back (C, X 40; D, X 100). PLEVA-like histopathologic features such as hyperkeratosis in the epidermis, mild acanthosis, elongated rete ridges, vacuolization of the basal layer with exocytosis of inflammatory cells, lymphohistiocytic infiltration around the vascular plexus and red blood cells (RBC) extravasation in the dermis were noticed in the H\&E staining figures.

Abbreviation: PLEVA, pityriasis lichenoides et varioliformis acuta.

patient was presumptively diagnosed as PLEVA and treated both locally (sulfur ointment, halometasone cream and black light therapy with long-wave ultraviolet light) and systemically (compound glycyrrhizin tablets and antihistamines) with no dermatologic improvement.

Three days after hospitalization, the patient presented with a $37.5^{\circ} \mathrm{C}$ fever and neurological manifestations including headache and nausea. Other neurological symptoms, such as dementia, personality change, mental confusion, memory impairments, euphoric mood, mania, paranoia and motor dysphasia did not present in this case. Her cerebral magnetic resonance imaging result was normal. Serological tests for HIV and hepatitis $\mathrm{C}$ virus antibodies were negative but positive for syphilis antibodies. Further examinations showed positive result of serum Treponema pallidum particle agglutination (TPPA) test (titer $\geq 1: 80$ ) but negative result of toluidine red unheated serum test (TRUST). The next day, the serumTPPA test was repeated again and remained positive (titer $\geq 1: 80$ ); Besides, a positive TRUST test with titer of $1: 512$ was presented after gradient dilution of serum.

Because the patient was suspected to have neurosyphilis, it was arranged for her to undergo a lumbar puncture. Cerebrospinal fluid (CSF) examinations showed normal results except CSF-TPPA test was positive (titer $\geq 1: 80$ ) and micro-total protein was decreased $(0.011 \mathrm{~g} / \mathrm{L})$. The patient admitted sexual intercourse with one male within the past seven months. Congenital syphilis was excluded because serum-TPPA test and serum-TURST test of her parents were negative. Physical examination was performed again and two rice-sized superficial ulcers were noticed on the labium majus area.

Considering the general and dermatological condition of the patient and lack of response to PLEVA treatment, the patient was treated with neurosyphilis therapy: aqueous crystalline penicillin G 24 million units IV daily for 14 days, followed with benzathine penicillin G 2.4 million units IM weekly for three weeks. After completing the therapy, the neurological and skin manifestations of the patient were significantly improved (Figure 3A-F) and serum-TRUST titer declined to 1:64 at December 19, 2016 and to 1:32 two months later. Based on all examination results and treatment response, the patient was ultimately diagnosed with secondary syphilis with probable neurosyphilis.

\section{Discussion}

As an important component of diagnosis, physical examination of the clinical presentations of a patient is critical 

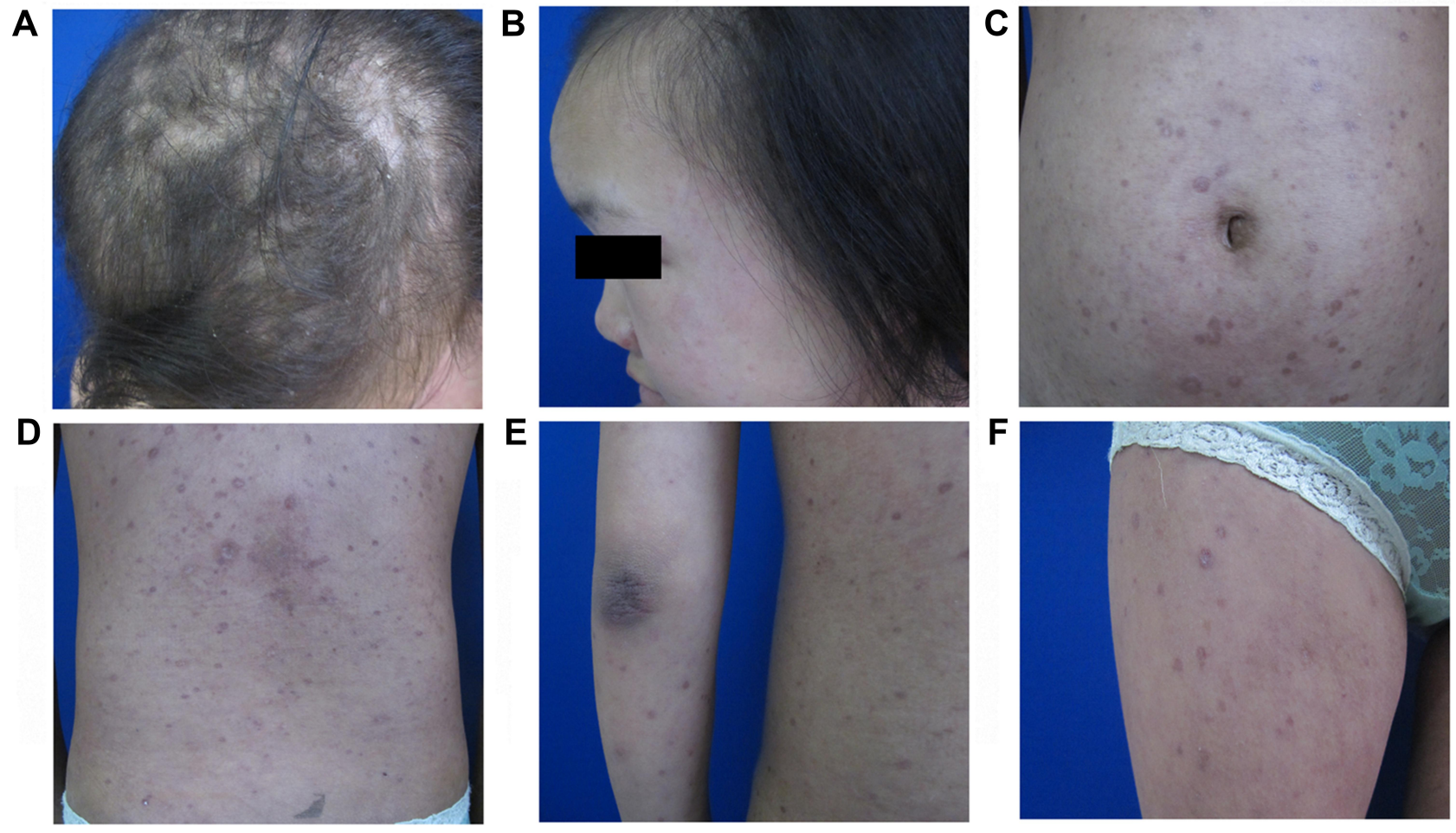

Figure 3 Clinical manifestations after neurosyphilis therapy. Erythema and papules on the scalp (A), face (B), trunk $(\mathbf{C}$ and $\mathbf{D})$ and extremities $(\mathbf{E}$ and $\mathbf{F})$ were significantly improved after neurosyphilis therapy treatment.

for the early diagnosis of secondary syphilis. Usually, generalized papulosquamous eruption accompanied by mucosal involvement is the most common manifestation of secondary syphilis; other clinical presentations including macular, papular, nodular, maculopapular, papulosquamous, lichenoid, pustular, crusted necrotic, ulcerative and annular lesions may also be observed in this disease. ${ }^{2}$ Secondary syphilis is very polymorphous and may mimic many other cutaneous diseases including pityriasis lichenoides (PL). ${ }^{1} \mathrm{PL}$ mainly appears in two forms, the acute form PLEVA and the chronic form pityriasis lichenoides chronica (PLC). PLEVA is a rare cutaneous disease manifested by recurrent eruptions of papules, which is said to be most common in children and young adults. ${ }^{3}$ Only two cases of PLEVA-like secondary syphilis have been reported, one in a 33-year-old HIV-infected patient ${ }^{4}$ and another in a 44-year-old HIV-negative patient. ${ }^{5}$ Similarly, a few published papers have reported secondary syphilis that mimics PLC. ${ }^{6,7}$ In the present case, we did not realize the possibility of syphilis at first because of the young age of the patient and the cutaneous manifestations such as relapsed systemic erythema and papules, necrotic crust and hyperpigmentation that were compatible with PLEVA, which hampered the diagnosis of this case.
Histopathologic examination is a "gold standard" for diagnosis of some diseases; however, just like the cutaneous manifestations of this disease, the histopathologic features of secondary syphilis are variable. In a study performed by Flamm et al., the authors retrospectively analyzed 106 biopsy specimens of secondary syphilis and found interstitial inflammation, endothelial swelling, irregular acanthosis and elongated slender rete ridges are the most common histopathologic features overall. They concluded that the independent value of many histologic features of syphilis may be overestimated. ${ }^{8}$ Secondary syphilis histologically has some overlapping features with PL. In another study performed by Flamm et al., the authors compared histopathologic features of secondary syphilis and PL. They found that there was no unique feature strongly associated with syphilis; therefore, it was difficult to distinguish syphilis from PL histologically. ${ }^{3}$ In the present case, the patient showed histological features such as acanthosis, interstitial inflammation and hyperkeratosis in the epidermis that have already been reported in PLEVA, thus further misleading the diagnosis of the case.

Secondary syphilis is usually confirmed by serologic tests and/or by direct detection of T. pallidum using immunohistochemistry or Warthin-Starry stain. Serologic tests, 
including treponemal tests such as TPPA, fluorescent treponemal antibody absorption (FTA-ABS) test and Treponema pallidum hemagglutination assay (TPHA) and nontreponemal tests such as venereal disease research laboratory (VDRL) test, rapid plasma reagin (RPR) test and TRUST test, seem to be sensitive in all stages of syphilis and thus can be generally used. ${ }^{9}$ In the present case, we used TPPA and TRUST tests to confirm the diagnosis of secondary syphilis. During serologic tests, there is one point which needs attention, which is that our serum-TRUST test was negative at first but positive after gradient dilution of serum, which is caused by the prozone phenomenon. During serologic tests, the prozone phenomenon, which occurs in high-titer specimens, should be fully considered by dilution samples to avoid false negative results. ${ }^{10,11}$ In addition to serologic tests, detection of spirochetes in skin tissues using immunohistochemistry or Warthin-Starry stain is helpful to confirm the diagnosis of secondary syphilis. Immunohistochemistry has better sensitivity and specificity than Warthin-Starry stain and thus can be widely used for secondary syphilis. ${ }^{12,13}$ In the present case, we did not perform this staining because our serologic tests were positive for syphilis.

Neurosyphilis is a severe consequence of syphilis and can occur at any stage of infection. The diagnosis of neurosyphilis is complex and currently based on clinical manifestations and results from serologic and CSF tests. In the present case, the patient was diagnosed as probable neurosyphilis according to the criteria of a Chinese expert consensus ${ }^{14}$ because she had a sexual life history before the outburst of cutaneous manifestations (with epidemiological history) and neurological symptoms such as headache and nausea (clinical features), and her CSF-TPPA test was positive (titer $\geq 1: 80$ ). Considering neurosyphilis can occur at any stage of infection and has serious consequences, we therefore used neurosyphilis therapy to treat this patient and achieved satisfactory results.

\section{Conclusion}

PLEVA clinically and histologically has some overlapping features with secondary syphilis. Physicians should be alert for the possibility of syphilis when encountering cases with PLEVA-like presentations but lack of response to PLEVA treatment, even though it is rare.

\section{Ethics Statement}

The publications of images were included in the guardian's consent for publication of the case. No ethical committee approval was required because the data were analyzed in a retrospective manner.

\section{Consent Statement}

Informed consent was provided by the patient's guardian for publication of the case.

\section{Disclosure}

The authors have no conflicts of interest to declare.

\section{References}

1. Çakmak SK, Tamer E, Karadağ AS, Waugh M. Syphilis: a great imitator. Clin Dermatol. 2019;37(3):182-191. doi:10.1016/j. clindermatol.2019.01.007

2. Wöhrl S, Geusau A. Clinical update: syphilis in adults. Lancet. 2007;369(9577):1912-1914. doi:10.1016/S0140-6736(07)60895-2

3. Flamm A, Alcocer VM, Kazlouskaya V, Kwon EJ, Elston D. Histopathologic features distinguishing secondary syphilis from its mimickers. J Am Acad Dermatol. 2020;82(1):156-160. doi:10.1016/j. jaad.2019.07.011

4. Pastuszczak M, Woźniak W, Jaworek AK, Wojas-Pelc A. Pityriasis lichenoides-like secondary syphilis and neurosyphilis in a HIV-infected patient. Postep Derm Alergol. 2013;30(2):127-130. doi:10.5114/pdia.2013.34166

5. Jacobson MA, Pollack RB, Maize JC Jr. Papulonecrotic eruption in a 44-year-old-man. South Med J. 2007;100(12):1221-1222. doi:10.1097/smj.0b013e31815a93bc

6. Lee EH, Lee SE, Kim DH, Yoon MS, Kim SC. Demonstration of Treponema pallidum by immunohistochemistry in a patient with secondary syphilis mimicking pityriasis lichenoides chronica. $J \quad$ Dermatol. 2012;39(6):567-568. doi:10.1111/j.13468138.2011.01436.x

7. Dar NR, Ali L, Nawaz MA, Mirza IA. Secondary syphilis lesions resembling pityriasis lichenoides. J Coll Physicians Surg Pak. 2007;17(11):689-690. doi:11.2007/JCPSP.689690

8. Flamm A, Parikh K, Xie Q, Kwon EJ, Elston DM. Histologic features of secondary syphilis: a multicenter retrospective review. $J$ Am Acad Dermatol. 2015;73(6):1025-1030. doi:10.1016/j. jaad.2015.08.062

9. Son JH, Park SY, Chung BY, Kim HO, Cho HJ, Park CW. Nodular secondary syphilis in an immunocompetent woman: case report and literature review. Dermatol Sin. 2018;36(1):36-41. doi:10.1016/j. dsi.2016.10.006

10. Liu LL, Lin LR, Tong ML, et al. Incidence and risk factors for the prozone phenomenon in serologic testing for syphilis in a large Cohort. Clin Infect Dis. 2014;59(3):384-389. doi:10.1093/cid/ciu325

11. Post JJ, Khor C, Furner V, Smith DE, Whybin LR, Robertson PW. Case report and evaluation of the frequency of the prozone phenomenon in syphilis serology-an infrequent but important laboratory phenomenon. Sex Health. 2012;9:488-490. doi:10.1071/SH11156

12. Fernandez-Nieto D, Ortega-Quijano D, Jimenez-Cauhe J, FernandezGonzalez P, Moreno-Moreno E, Bea-Ardebol S. Secondary syphilis mimicking tuberculoid leprosy in an HIV-positive individual: a case report. Int J Std Aids. 2019;30(12):1235-1238. doi:10.1177/ 0956462419869136 
13. Liu HF, Goh BT, Huang TY, et al. Secondary syphilis presenting as erythema multiforme in a HIV-positive homosexual man: acase report and literature review. Int J Std Aids. 2018;30(3):304-309. doi: $10.1177 / 0956462418805197$
14. Guo L. 2019 Chinese expert consensus statement on diagnosis and treatment of syphilis. Chin Med J. 2020;133(19):2335-2337. doi:10.1097/CM9.0000000000001035

\section{Publish your work in this journal}

Clinical, Cosmetic and Investigational Dermatology is an international, peer-reviewed, open access, online journal that focuses on the latest clinical and experimental research in all aspects of skin disease and cosmetic interventions. This journal is indexed on CAS.

Submit your manuscript here: https://www.dovepress.com/clinical-cosmetic-and-investigational-dermatology-journal
The manuscript management system is completely online and includes a very quick and fair peer-review system, which is all easy to use. Visit http://www.dovepress.com/testimonials.php to read real quotes from published authors. 